BULL. AUSTRAL. MATH. SOC.

VOL. 34 (1986) $321-334$

\title{
THE HIGHER DERIVATIVES OF FUNCTIONS BOUNDED IN VARIOUS SENSES
}

\author{
SHINJI YAMASHITA
}

An extension (Theorem 1) of Schwarz and Pick's lemma motivates us to study the analogues for functions which are bounded in the sense of Bloch, normal, or Yoshida. A typical result is that, for a function $f$ holomorphic in $D=\{|z|<1\}$ and Bloch, that is, $\alpha=\sup \left(1-|z|^{2}\right) \times\left|f^{\prime}(z)\right|<\infty$, with the expansion $f(w)=$ $z \in D$

$c_{0}+c_{n}(w-z)^{n}+\ldots(n \geqq 1)$ about $z \in D$, we have $\left(1-|z|^{2}\right)^{n}\left|f^{(n)}(z)\right| / n ! \leqq A_{n}^{\alpha}$, where $A_{n}$ is an absclute constant; the estimate is sharp.

\section{Introduction.}

The celebrated Schwarz-Pick lemma on functions $f$ holomorphic and bounded, $|f|<1$, in the disk $D=\{|z|<1\}$, states that

$$
\left(1-|z|^{2}\right)\left|f^{\prime}(z)\right| /\left(1-|f(z)|^{2}\right) \leqq 1, \quad z \in D \text {. }
$$

our researches begin with the following extension.

Received 5 December 1985.

Copyright Clearance Centre, Inc. Serial-fee code: 0004-9727/86 $\$ A 2.00+0.00$. 
THEOREM 1. Let $f$ be a function holomorphic and bounded, $|f|<1$, in $D$, and let $z \in D$. Suppose that

$$
f(w)=c_{0}+c_{n}(w-z)^{n}+c_{n+1}(w-z)^{n+1}+\ldots
$$

in a neighbourhood of $z$, where $n \geqq 1$ depends on $z$ and $c_{n}=0$ is possible. Then,

$$
\left(1-|z|^{2}\right)^{n}\left|f^{(n)}(z)\right| /\left\{n !\left(1-|f(z)|^{2}\right)\right\} \leqq 1 \text {. }
$$

The inequality (1.3) is sharp in the sense that equality holds for the function

$$
\text { (1.4) } \quad f(w)=e^{i a}\{(w-z) /(1-\bar{z} w)\}^{n} \quad(a \text {; a real constant })
$$

of $w$. Theorem 1 extends the Schwarz-Pick lemma because (1.1) is the case $n=1$ in Theorem 1 .

Are similar results true for functions meromorphic or holomorphic in $D$ or in $\mathbb{C}=\{|z|<\infty\}$ if they are bounded in appropriate senses?

For positive answers we consider three kinds of boundedness, namely, Bloch, normal, and Yosida, which are explained below.

THEOREM 2. Let $f$ be a fronction holomorphic in $D$ with

$$
0<\alpha=\alpha(f) \equiv \sup _{z \in D}\left(1-|z|^{2}\right)\left|f^{\prime}(z)\right|<\infty .
$$

Suppose (1.2) also holds for this $f$. Then,

$$
\left(1-|z|^{2}\right)^{n}\left|f^{(n)}(z)\right| / n ! \leqq A_{n}^{\alpha} \text {, }
$$

where

$$
\begin{aligned}
A_{n} & =1, & \text { if } n=1, \\
& =\frac{n+1}{2 n}\left(1+\frac{2}{n-1}\right)^{\frac{n-1}{2}}, & \text { if } n>1 .
\end{aligned}
$$

The inequality (1.6) is sharp in the sense that the equaltiy holds for the $f$ of (1.4). We call $f$ Bloch if (1.5) is satisfied [3]. The case $n=1$ is trivial. The sequence $\left\{A_{n}\right\}$ is increasing and tends to $e / 2$, so that $A_{n}<e / 2=1.359 \ldots$ A calculation yields that for the function $w^{n}$ of $w \in D, A_{n}=1 / \alpha\left(w^{n}\right)$. 
To deal with a meromorphic function $f$ in $D$ or in $C$, we write for $n \geqq 1$, and for a variable $z$,

$$
\begin{aligned}
f_{n}^{\#}(z) & =\left|f^{(n)}(z)\right| /\left\{n !\left(1+|f(z)|^{2}\right)\right\}, & & \text { if } \quad f(z) \neq \infty ; \\
& =(1 / f)_{n}^{\#}(n), & & \text { if } \quad f(z)=\infty .
\end{aligned}
$$

In particular, if (1.2) holds for this $f$ at $z$ where $f(z) \neq \infty$, then $f_{n}^{\prime \prime}(z)=\left|c_{n}\right| /\left(1+\left|c_{0}\right|^{2}\right)$, while if $f(z)=\infty$ with

$$
1 / f(w)=p_{n}(w-z)^{n}+p_{n+1}(w-z)^{n+1}+\ldots
$$

in a neighbourhood of $z$, then $(1 / f)_{n}^{\#}(z)=\left|p_{n}\right| \geqq 0$.

The quantity $f_{l}^{\#}(z)$ is known as the spherical derivative of $f$ at $z$; this is a continuous function of $z \in D$.

THEOREM 3. Let $f$ be a function meromorphic in $D$ with

$$
0<\beta=\beta(f) \equiv \sup _{z \in D}\left(1-|z|^{2}\right) f_{I}^{*}(z)<\infty .
$$

Suppose that (1.2) or (1.7) holds in a neighbourhood of $z \in D$ with $n \geqq 1$ for this $f$. Then,

$$
\left(1-|z|^{2}\right)^{n} f_{n}^{\#}(z) \leqq B_{n}(\beta)
$$

where

$$
B_{n}(\beta)=\inf _{0<x<\pi /(2 \beta)}(\tan \beta x) /(\tanh x)^{n}
$$

We have no information on the sharpness of (1.9). We call $f$ normal if (1.8) is satisfied [2]. Computations yield that $B_{1}(\beta)=\beta$ (thus, the case $n=1$ is trivial) and the infimum in (1.10) for $n>1$ is attained at the root $x$ of the equation

$$
\beta \sinh (2 x)-n \sin (2 \beta x)=0
$$

lying in $0<x<\pi /(2 \beta)$.

THEOREM 4. Let $f$ be a function meromorphic in $\mathbb{C}$ with

$$
0<\gamma=\gamma(f) \equiv \sup _{z \in \mathbb{C}} f_{1}^{\#}(z)<\infty .
$$


Suppose that (1.2) or (1.7) holds in a neighbourhood of $z \in D$ with $n \geqq 1$ for this $f$. Then,

$$
f_{n}^{\#}(z) \leqq c_{n} \gamma^{n}
$$

where

$$
c_{n}=\inf _{0<x<\pi / 2}(\tan x) / x^{n}
$$

We have no information on the sharpness of the estimate (1.13). We call $f$ Yosida if (1.12) is satisfied; this is equivalent to saying that $f$ is of class (A) in K. Yosida's sense [6]. The case $n=1$ is trivial because $C_{1}=1$. The infimum in (1.14) for $n>1$ is attained at the root $x$ of the equation

$$
2 x-n \sin (2 x)=0
$$

in $0<x<\pi / 2$. Computations yield

$$
\begin{aligned}
& C_{2}=1.54 \ldots ; C_{3}=1.46 \ldots ; C_{4}=1.23 \ldots ; C_{5}=0.97 \ldots ; \\
& C_{6}=0.73 \ldots ; C_{7}=0.54 \ldots ; C_{8}=0.39 \ldots ; \text { and so on. }
\end{aligned}
$$

Hitherto we have been concerned with $f$ at $z$ under the restriction (1.2) or (1.7). We shall later estimate the left-hand sides of (1.3), (1.6), (1.9) and (1.13) without the cited restriction. The results are, however, not complete enough in the sense that we have no information on the sharpness of the bounds.

\section{Proof of Theorem 1 .}

We note first that if $h$ is holomorphic and bounded, $|h|<1$, in $D$, then $\left|h^{(n)}(0)\right| \leqq n !$. This follows from the Cauchy formula for $h^{(n)}(0)$ expressed by the integral on the circle $\{|w|=r\}$. This gives $\left|h^{(n)}(0)\right| \leqq n ! r^{-n}, 0<r<1$, and letting $r \rightarrow 1$ we arrive at the inequality.

LEMMA 2.1. Let $f$ be meromorphic in $D$ and suppose that $f(z) \neq \infty$ at a point $z \in D$. Let
(2.1)

$$
g(w)=f((w+z) /(1+\bar{z} w)),
$$$$
w \in D
$$

Then for $n \geqq 1$, 
(2.2) $\left(1-|z|^{2}\right)^{n} f^{(n)}(z) / n !=\sum_{k=0}^{n-1}\left(\begin{array}{c}n-1 \\ k\end{array}\right) z^{-k} g^{(n-k)}(0) /(n-k) !$.

This lemma for meromorphic functions will also frequently be used later. o. Szász [4, p. 307] obtained (2.2) for $f$ pole-free in $D$. His method of proof does not carry over to meromorphic $f$ and must be modified.

For small $r, 0<r<1$, the Cauchy formula reads:

$$
f^{(n)}(z)=\frac{n !}{2 \pi i} \int_{|\zeta-z| /|1-z \zeta|=r} \frac{f(\zeta)}{(\zeta-z)^{n+1}} d \zeta \text {. }
$$

Consequently, on setting $w=(\zeta-z) /(1-\overline{z \zeta})$, we have

$$
f^{(n)}(z)=\frac{n !}{2 \pi i\left(1-|z|^{2}\right)^{n}} \int_{|w|=x} g(w) w^{-n-1}(1+\bar{z} w)^{n-1} d w .
$$

Substituting the identities

$$
g(w) w^{-n-1}=\sum_{k=0}^{\infty} \frac{g^{(k)}(0)}{k !} w^{k-n-1}
$$

and

$$
(1+\bar{z} w)^{n-1}=\sum_{k=0}^{n-1}\left(\begin{array}{c}
n-1 \\
k
\end{array}\right) \bar{z}^{-k} w^{k}
$$

in (2.3) we have (2.2).

For the proof of Theorem 1 we consider $g$ of (2.1) for the bounded $f$ of Theorem 1 , and further we define

$$
F(w)=\{g(w)-g(0)\} /\{1-\overline{g(0)} g(w)\}, \quad w \in D .
$$

On considering the $n$-th dexivatives of both sides of

$$
F(w)-\overline{g(0)} g(w) F(w)=g(w)-g(0)
$$

at $w=0$ we obtain

$$
F^{(n)}(0)-\overline{g(0)} \sum_{k=0}^{n}\left(\begin{array}{l}
n \\
k
\end{array}\right) g^{(k)}(0) F^{(n-k)}(0)=g^{(n)}(0) \text {. }
$$

Since $g(0)=f(z), g^{(k)}(0)=0$ for $1 \leqq k \leqq n-1$ for $n \geqq 2$, it follows from (2.2) that $g^{(n)}(0)=\left(1-|z|^{2}\right)^{n} f^{(n)}(z)$. Therefore,

$$
F^{(n)}(0)=\frac{g^{(n)}(0)}{1-|g(0)|^{2}}=\frac{\left(1-|z|^{2}\right)^{n} f^{(n)}(z)}{1-|f(z)|^{2}},
$$


which, together with $\left|F^{(n)}(0)\right| \leqq n !$, yields (1.3).

For the function $f$ of (1.4) we have $g(w)=e^{i a w^{n}}=F(w)$. Since $n !=\left|F^{(n)}(0)\right|$, we have equality in (1.3).

Remark. One might suspect that (1.3) holds for each $n \geqq 2$ and at each $z \in D$ without the restriction (1.2). A counterexample is supplied by $f(w)=w^{n+k}(n \geqq 2)$ for sufficiently large $k$. Since

$$
f^{(n)}(w)=Q w^{k}, \quad Q=(n+k)(n+k-1) \cdots(k+1)>k^{n},
$$

it follows that, on the circle

$$
|w|=r_{k} \equiv\left(1+\frac{2 n}{k}\right)^{-1 / 2},
$$

we have

$$
\left(1-|w|^{2}\right)^{n}\left|f^{(n)}(w)\right|=\frac{Q\left(\frac{2 n}{k}\right)^{n}}{\left(1+\frac{2 n}{k}\right)^{k / 2+n}}>\frac{(2 n)^{n}}{\left(1+\frac{2 n}{k}\right)^{k / 2+n}} .
$$

Therefore

$$
\lim _{k \rightarrow \infty} \inf \left\{\min \left(1-|w|^{2}\right)^{n}\left|f^{(n)}(w)\right|\right\} \geqq(2 n)^{n} / e^{n}>n ! .
$$

Accordingly, there exists $k>1$ such that

$$
\left(1-|w|^{2}\right)^{n}\left|f^{(n)}(w)\right| /\left\{n !\left(1-|f(w)|^{2}\right)\right\}>1
$$

on the circle $|w|=r_{k}$. Since $f^{\prime}(w) \neq 0$ at each point of $|w|=r_{k}$, this violates (1.3) if (1.2) is dropped.

3. Removal of condition (1.2) in Theorem 1 .

If we do not assume the restriction (1.2) at $z$, then we have

THEOREM 5. Let $f$ be a function holomorphic and bounded, $|f|<1$, in $D$. Then, for each $n \geqq 1$, and at each $z \in D$, we have

$$
\begin{aligned}
(1 & \left.-|z|^{2}\right)^{n}\left|f^{(n)}(z)\right| /\left\{n !\left(1-|f(z)|^{2}\right)\right\} \leqq \\
& \leqq \\
& (1+|z|+|f(z)|)^{n-1} \leqq 3^{n-1} .
\end{aligned}
$$

Again, Theorem 5 yields (1.1) in the case $n=1$. As in the proof of Theorem 1 we observe $g$ and $F$ in (2.4). It then follows from (2.5) that 


$$
\frac{F^{(k)}(0)}{k !}-\overline{f(z)} \sum_{j=0}^{k} \frac{g^{(j)}(0)}{j !} \frac{F^{(k-j)}(0)}{(k-j) !}=\frac{q^{(k)}(0)}{k !}, \quad k \geqq 1 .
$$

Since $F(0)=0$ and $\left|F^{(\nu)}(0) / v !\right| \leqq 1, v \geqq 1$, it follows that

$$
\left|\frac{g^{(k)}(0)}{k !}\right| \leqq 1-|f(z)|^{2}+|f(z)| \sum_{j=1}^{k-1}\left|\frac{g^{(j)}(0)}{j !}\right|, \quad k \geqq 1 .
$$

Induction on (3.2) now shows that

$$
\left|\frac{g^{(k)}(0)}{k !}\right| \leqq\left(1-|f(z)|^{2}\right)(1+|f(z)|)^{k-1}, k \geqq 1,
$$

which, combined with (2.2), yields (3.1).

\section{Proof of Theorem 2.}

We begin with

LEMMA 4.1. If $g$ is Bloch, then

$$
\left|g^{(n)}(0)\right| \leqq n ! A_{n}^{\alpha}(g) \quad(n \geqq 1) .
$$

For the proof we may suppose that $n>1$. Then, for $0<r<1$,

$$
\begin{aligned}
\left|g^{(n)}(0)\right| & =\left|\left(g^{\prime}\right)^{(n-1)}(0)\right| \\
& =\left|\frac{(n-1) !}{2 \pi} \int_{0}^{2 \pi} \frac{g^{\prime}\left(r e^{i t}\right)}{r^{n-1} e^{i(n-1) t}} d t\right| \leqq n ! \alpha(g) \phi(r),
\end{aligned}
$$

where

$$
1 / \phi(r)=n r^{n-1}\left(1-r^{2}\right) \text {. }
$$

The function $\phi$ has the minimum $A_{n}$ at $v=\{(n-1) /(n+1)\}^{1 / 2}$, which completes the proof of (4.1).

For the proof of Theorem 2 we consider $g$ of (2.1) for our pole-free $f$. Since $\alpha=\alpha(f)=\alpha(g)$, and since

$$
g^{(n)}(0)=\left(1-|z|^{2}\right)^{n} f^{(n)}(z),
$$

it follows from Lemma 4.1, applied to this $g$, that (1.6) holds.

For the sharpness of $(1.6)$ we note that $g(w)=e^{i a_{w} n}$ for the $f$ of (1.4). Then,

$$
n !=\left|g^{(n)}(0)\right|=\left(1-|z|^{2}\right)^{n}\left|f^{(n)}(z)\right| \text {, }
$$

while

$$
\alpha(f)=\alpha\left(w^{n}\right)=1 / A_{n} \text {. }
$$

These show that equality holds in (1.6) for the $f$ of (1.4). 
5. Removal of condition (1.2) in Theorem 2 . If we remove the restriction (1.2) at $z$, then we have

THEOREM 6. Let $f$ be a function holomorphic in $D$ satisfying (1.5). Then, for each $n \geqq 1$ and at each $z \in D$, we have (5.1) $\quad\left(1-|z|^{2}\right)^{n}\left|f^{(n)}(z)\right| / n ! \leqq \alpha_{k=0}^{n-1}\left(\begin{array}{c}n-1 \\ k\end{array}\right)|z|^{k} A_{n-k} \leqq A_{n}^{\prime \alpha}$, where $\quad A_{n}^{\prime}={ }_{k=0}^{n_{\bar{\Sigma}}^{1}}\left(\begin{array}{c}n-1 \\ k\end{array}\right) A_{n-k}$.

For the proof of (5.1) we have only to combine (2.2) with Lemma 4.1.

6. Proofs of Theorems 3 and 4 .

For the proof of Theorem 3 we may assume that $f(z) \neq \infty$ and $f$ has the expansion (1.2) near $z$. The case (1.7) is handled similaxly by considering $1 / f$ instead of $f$ with $B(f)=B(1 / f)$.

Consider $g$ of (2.1) for this $f$ and set

$$
F(w)=\{g(w)-g(0)\} /\{1+\overline{g(0)} g(w)\}, \quad w \in D .
$$

Then $\beta(F)=\beta(g)=\beta(f)$ yields that

$$
F_{1}^{\#}(\zeta)|d \zeta| \leqq B\left(1-|\zeta|^{2}\right)^{-1}|d \zeta|, \quad \zeta \in D \text {. }
$$

On integrating from 0 to $w$ along the radius of $D$, we have

$$
\tan ^{-1}|F(w)| \leqq \beta \tanh ^{-1}|w|<K \quad(0<K<\pi / 2),
$$

if $|w|<\tanh (K / \beta) \equiv R$. The left-most quantity in (6.1) is the length of the arc on the great circle (the meridian) between 0 and $F(w)$ on the Riemann sphere.

The function $h(w)=F(R w) / \tan K$ of $w \in D$ is then bounded, $|h|<1$, so that $\left|h^{(n)}(0)\right| \leqq n !$, or,

$$
\left|F^{(n)}(0)\right| \leq n !(\tan K) / R^{n} \text {. }
$$

It now follows from

$$
F^{(n)}(0)+\overline{g(0)} \sum_{k=0}^{n}\left(k_{k}^{n}\right) g^{(k)}(0) F^{(n-k)}(0)=g^{(n)}(0),
$$

together with $g(0)=f(z), g^{(k)}(0)=0$ for $1 \leqq k \leqq n-1$ for $n \geqq 2$, and $g^{(n)}(0)=\left(1-|z|^{2}\right)^{n} f^{(n)}(z)$ resulting from (2.2), that

$$
\left(1+|f(z)|^{2}\right) F^{(n)}(0)=g^{(n)}(0)=\left(1-|z|^{2}\right)^{n} f^{(n)}(z) .
$$

Therefore, (1.9) follows from (6.2) on setting $x=K / B$ and on taking the infimum. 
The function $\Phi(x)=(\tan \beta x) /(\tanh x)^{n}, \quad 0<x<\pi /(2 \beta)$, is increasing for $n=1$. If $n>1$, then the sign of $\Phi$ is the same as that of

$$
\Psi(x)=\sinh (2 x)-(n / \beta) \sin (2 \beta x) \equiv \lambda(x)-\mu(x) .
$$

The graph $y=\lambda(x)$ has tangent $y=2 x$ at the origin, is convex downwards, and increasing, while the graph $y=\mu(x)$ has tangent $y=2 n x$ at the origin and is convex upwards. Since $\Psi(\pi /(2 \beta))=\sinh (\pi / \beta)>0$, it follows that (1.11) holds only at one point $x \in(0, \pi /(2 \beta))$.

For the proof of Theorem 4 we set

$$
g(w)=f(w+z) \text { and } F(w)=\{g(w)-g(0)\} /\{1+\overline{g(0)} g(w)\},
$$

$w \in \Phi$. Then, $\gamma(F)=\gamma(g)=\gamma(f)$, and, this time, (6.1) becomes

$$
\tan ^{-1}|F(w)| \leqq \gamma|w|<K \quad(0<K<\pi / 2) \text {, }
$$

if $|\omega|<K / \gamma \equiv R$. Observing that

$$
\left(1+|f(z)|^{2}\right) F^{(n)}(0)=g^{(n)}(0)=f^{(n)}(z)
$$

in the case (1.2) for this $f$, we deduce (1.13) from

$$
\left|F^{(n)}(0)\right| \leqq n !(\tan K) / R^{n}=n ! \gamma^{n}(\tan K) / K^{n} \text {. }
$$

The case (1.7) is treated similarly by considering $1 / f$.

The sign of the derivative of the function $(\tan x) / x^{n}$ is the same as that of $2 x-n \sin (2 x)$ for $0<x<\pi / 2$, so that, the minimum in the case $n>1$ is attained at the root of (1.15).

7. Meromorphic functions with neither condition (1.2) nor (1.7).

We begin with a counterpart of Theorem 3 .

THEOREM 7. Let $f$ be a function meromorphic in $D$ satisfying (1.8). Then, for each $n \geqq 1$ and at each $z \in D$ we have

(7.1) $\quad\left(1-|z|^{2}\right)^{n} f_{n}^{\#}(z) \leqq$

$$
\begin{gathered}
\inf _{0<x<\pi / 2 \beta)} \frac{\tan \beta x}{(\tanh x)^{n}}(1+|z| \tanh x+|f(z)| \tan \beta x)^{n-1}, \\
\text { if } f(z) \neq \infty ;
\end{gathered}
$$

(7.2) $\left(1-|z|^{2}\right)^{n} f_{n}^{\#}(z) \leqq \inf _{0<x<\pi /(2 \beta)} \frac{\tan \beta x}{(\tanh x)^{n}}(1+|z| \tanh x)^{n-1}$,

$$
\text { if } f(z)=\infty \text {. }
$$


On replacing $f$ in (7.1) by $1 / f$, we obtain (7.2). For $x=\pi /(4 \beta)$ we also have, for the function in the right-hand side of (7.1) ,

$$
\text { (7.1) } \quad\left(1-|z|^{2}\right)^{n} f_{n}^{\#}(z) \leqq \Gamma^{-n}(1+|z| \Gamma+|f(z)|)^{n-1}, \quad f(z) \neq \infty \text {, }
$$

where $\Gamma=\tanh \{\pi /(4 \beta)\}$.

For the proof of Theorem 7 we let $g$ and $F$ be as in the proof of Theorem 3; we may assume that $f(z) \neq \infty$. It then follows from

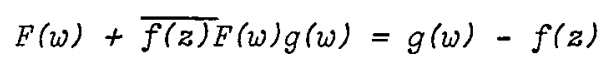

that

(7. 3) $\frac{E^{(k)}(0)}{k !}+\overline{f(z)} \sum_{j=0}^{k-1} \frac{g^{(j)}(0)}{j !} \frac{E^{(k-j)}(0)}{(k-j) !}=\frac{g^{(k)}(0)}{k !}, k \geqq 1$,

because $F(0)=0$. By the same reasoning as in the proof of (6.2) we have, for each $\ell \geqq 1$,

$$
\left|F^{(\ell)}(0) / \ell !\right| \leqq \tan K / R^{\ell} \text { for } R=\tanh (K / \beta), 0<K<\pi / 2 \text {. }
$$

It then follows from (7.3), together with $g(0)=f(z)$ that, for $k \geqq 1$,

$$
\text { (7.4) }\left|\frac{g^{(k)}(0)}{k !}\right| \leqq\left(1+|f(z)|^{2}\right) \frac{\tan K}{R^{k}}+|f(z)| \sum_{j=1}^{k-1} \frac{\tan K}{k-j}\left|\frac{g^{(j)}(0)}{j !}\right| \text {. }
$$

Induction on (7.4) now gives

$$
\left|\frac{g^{(k)}(0)}{k !}\right| \leq \frac{\tan K}{R^{k}}\left(1+|f(z)|^{2}\right)(1+|f(z)| \tan K)^{k-1}, k \geqq 1 \text {. }
$$

Combining (2.2) with (7.5), and setting $x=K / B$, we obtain (7.1).

THEOREM 8. Let $f$ be a function meromorphic in $\$$ with (1.12). Then, for each $n \geqq 1$ and at each $z \in \mathbb{C}$ we have

$$
\begin{gathered}
f_{n}^{\#}(z) \leqq \gamma^{n} \inf _{\substack{0<x<\pi / 2 \\
x^{n}}} \frac{\tan x}{1+|f(z)| \tan x)^{n-1},} \\
\text { if } f(z) \neq \infty ;
\end{gathered}
$$

(7.7) $f_{n}^{\#}(z) \leqq \gamma^{n} C_{n}$, if $f(z)=\infty$,

where $C_{n}$ is the same as in (1.14). 
On replacing $f$ in (7.6) by $1 / f$, we obtain (7.7), We emphasize that (1.13) in case $f(z)=\infty$ is ameliorated:

If $f(z)=0$ or $f(z)=\infty$, then, for each $v \geqq 1$,

$$
f_{v}^{\#}(z) \leqq C_{v} \gamma^{\nu} \text {. }
$$

In effect, the case $f(z)=0$ follows from $(7.6)$, while the case $f(z)=\infty$ is $(7.7)$.

On substituting $x=\pi / 4$ in the function in the right-hand side of (7.6) we have

$$
f_{n}^{\#}(z) \leqq(4 \gamma / \pi)^{n}(1+|f(z)|)^{n-1} \text {, if } f(z) \neq \infty .
$$

For the proof of Theorem 8 we may assume that $f(z) \neq \infty$. Let $g$ and $F$ be as in the proof of Theorem 4 . Since

$$
|F(w)| \leqq \tan K \quad \text { for } \quad|\omega|<K / \gamma \equiv R \quad(0<K<\pi / 2) \text {, }
$$

(7.5) for this $g$ and $R$ is true. In this case,

$$
\begin{gathered}
\left|f^{(n)}(z) / n !\right|=\left|g^{(n)}(0) / n !\right| \\
\leqq \gamma^{n} \frac{\tan K}{K^{n}}\left(1+|f(z)|^{2}\right)(1+|f(z)| \tan K)^{n-1},
\end{gathered}
$$

whence

$$
f_{n}^{\#}(z) \leqq \gamma^{n} \frac{\tan K}{K^{n}}(1+|f(z)| \tan K)^{n-1} .
$$

On setting $K=x$ and on taking the infimum we obtain (7.6) .

8. Concluding remarks.

For $f$ meromorphic and normal in $D$, with (1.8), the present author proved that

$$
\left(1-|z|^{2}\right)^{2} f_{1}^{\#}(z)\left(f^{\prime}\right)_{1}^{\#}(z)
$$

is bounded in $D$ [5, Theorem 1], and later, P. A. Lappan proved that the continuous function

$$
F_{n}(z)=\left(1-|z|^{2}\right)^{n} \prod_{k=0}^{n-1}\left(f^{(k)}\right)_{1}^{\#}(z)
$$

is bounded in $D$ for each $n>1$ [1, Theorem 1].

We shall find a concrete upper bound of $F_{n}$ in terms of $n>1$ and $\Gamma=\tanh \{\pi /(4 \beta)\}$ for nonconstant $f$, namely, 
(8.1) $\sup _{z \in D} F_{n}(z) \leqq n ! 2^{2-n} \Gamma^{-n}(2+\Gamma)^{n-1}\left\{2^{-1}+\sum_{k=1}^{n-1}[\Gamma /(2+\Gamma)]^{k} / k !\right\}$.

The situation is similar for $f$ meromorphic and Yosida in $\Phi$, with (1.12). For each $n>1$, the continuous function

$$
G_{n}(z)=\prod_{k=0}^{n-1}\left(f^{(k)}\right)_{1}^{\#}(z)
$$

is bounded in $\mathbb{\Phi}$, namely,

$$
\sup _{z \in \mathbb{Q}} G_{n}(z) \leqq n ! 2(4 \gamma / \pi)^{n}\left\{2^{-1}+\sum_{k=1}^{n-1}[\pi /(8 \gamma)]^{k} / k !\right\} \text {. }
$$

We begin with

LEMMA 8.1. [1, Lemma 2]. For $f$ meromorphic in $D$ or in $\$$, for each $n>1$, and for each $z$ where $f(z) \neq \infty$, we have

$$
\prod_{k=0}^{n-1}\left(f^{(k)}\right)_{1}^{\#}(z) \leqq n ! f_{n}^{\#}(z) \prod_{k=1}^{n-1} \max \left(2,\left|f^{(k)}(z)\right|\right) \text {. }
$$

LEMMA 8.2. For $f$ meromorphic in $D$ or in $\downarrow$, for each $n>1$, and for each $z$ where $f(z) \neq 0, \infty$, we have

$$
\begin{aligned}
& n ! f_{n}^{\#}(z) \prod_{k=1}^{n-1} \max \left(2,\left|f^{(k)}(z)\right|\right) \\
& \leqq n ! 2^{1-n}(1 / f)_{n}^{\#}(z)+\left|n ! 2^{2-n} / f(z)\right| \sum_{k=1}^{n-1}(1 / f)_{n-k}^{\#}(z) / k ! .
\end{aligned}
$$

Actually, applying the Leibniz rule to the identity $f \cdot(1 / f)=1$ near $z$, we obtain

$$
f^{(n)}(z)=-f(z) \sum_{k=0}^{n-1}\left(\begin{array}{l}
n \\
k
\end{array}\right) f^{(k)}(z)(1 / f)^{(n-k)}(z) .
$$

Therefore,

$$
\left|f^{(n)}(z)\right| \leqq|f(z)|^{2}\left|(1 / f)^{(n)}(z)\right|+|f(z)|_{k=1}^{n-1}\left(_{k}^{n}\right)\left|f^{(k)}(z)(1 / f)^{(n-k)}(z)\right|,
$$

whence

$$
\begin{aligned}
& n ! f_{n}^{\#}(z) \leqq\left|(1 / f)^{(n)}(z)\right| /\left(1+|1 / f(z)|^{2}\right)+ \\
& +|1 / f(z)|_{k=1}^{n-1}(n)\left|f^{(k)}(z)(1 / f)^{(n-k)}(z)\right| /\left(1+|1 / f(z)|^{2}\right) .
\end{aligned}
$$


On dividing both sides by

$$
\prod_{k=1}^{n-1} \max \left(2,\left|f^{(k)}(z)\right|\right)
$$

we obtain $(8,4)$.

For the proof of (8.1) we begin with the case $|f(z)| \leqq 1$. Then, (7.1)' yields that

$$
\left(1-|z|^{2}\right)^{n} f_{n}^{\#}(z) \leqq \Gamma^{-n}(2+\Gamma)^{n-1},
$$

whence, together with (8.3), we obtain

$$
F_{n}(z) \leqq n ! 2^{1-n} \Gamma^{-n}(2+\Gamma)^{n-1} \text {. }
$$

In the case $1<|f(z)|<\infty$, it follows from (7.1)' for $1 / f$ and $n-k(n>k \geqq 0)$ that

$$
\begin{aligned}
& \left(1-|z|^{2}\right)^{n}(1 / f)_{n-k}^{\#}(z) \leqq\left(1-|z|^{2}\right)^{n-k}(1 / f)_{n-k}^{\#}(z) \\
& \leq \Gamma^{-n+k}(2+\Gamma)^{n-k-1},
\end{aligned}
$$

which, together with (8.3) and (8.4), yields that

$$
F_{n}(z) \leqq n ! 2^{1-n} \Gamma^{-n}(2+\Gamma)^{n-1}+n ! 2^{2-n} \Gamma^{-n}(2+\Gamma)^{n-1^{n-1}} \sum_{k=1}^{n}\{\Gamma /(2+\Gamma)\}^{k} / k ! \text {. }
$$

Combining this with (8.5), and observing that $F_{n}$ is continuous in $D$, we have (8.1).

$$
\text { For the proof of (8.2), we first consider the case }|f(z)| \leqq 1 \text {. }
$$

Then, (7.6)' yields that

$$
f_{n}^{\#}(z) \leqq(4 \gamma / \pi)^{n} 2^{n-1},
$$

which, together with (8.3), asserts that

$$
G_{n}(z) \leqq n !(4 \gamma / \pi)^{n} \text {. }
$$

In the case $l<|f(z)|<\infty,(7.6)$, for $1 / f$ and for $n-k$ reads that

$$
(1 / f)_{n-k}^{\#}(z) \leqq(4 \gamma / \pi)^{n-k} 2^{n-k-1},
$$

which, together with (8.3) and (8.4), yields that

$$
G_{n}(z) \leqq n !(4 \gamma / \pi)^{n}+n ! 2(4 \gamma / \pi)^{n} \sum_{k=1}^{n-1}\{\pi /(8 \gamma)\}^{k} / k ! \text {. }
$$

Combining this with (8.6), and observing that $G_{n}$ is continuous in $\mathbb{C}$, 
we have (8.2).

Finally, we cannot expect a similar estimate for Bloch functions. Actually, for the Bloch function $f(z)=-\log (1-z)(f(0)=0)$ in $D$, we have $f^{(k)}(z)=(k-1) !(1-z)^{-k}(k>1)$. Therefore, for $0<x<1$ and for $n>1$,

$$
\left(1-x^{2}\right)^{n} \prod_{k=1}^{n} f^{(k)}(x)=\left(\prod_{k=1}^{n}(k-1) !\right)(1+x)^{n}(1-x)^{n(1-n) / 2},
$$

so that this tends to $\infty$ as $x \rightarrow 1-0$.

\section{References}

[1] P. A. Lappan, "The spherical derivative and normal functions", Ann. Acad. Sci. Fenn. Ser. A.I. Math. 3 (1977), 301-310.

[2] 0 . Lehto and K. I. Virtanen, "Boundary behaviour and normal meromorphic functions", Acta Math. 97 (1957), 47-65.

[3] C. Pommerenke, "On Bloch functions", J. London Math. Soc. (2), 2 (1970), 689-695.

[4] O. Szász, "Ungleichheitsbeziehungen für die Ableitungen einer Potenzreihe, die eine im Einheitskreise beschränkte Funktion darstellt", Math. 2. 8 (1920), 303-309.

[5] S. Yamashita, "On normal meromorphic functions", Math. 2. 141 (1975), 139-145.

[6] K. Yosida, "On a class of meromorphic functions", Proc. Phys.-Math. Soc. Jcp. 16 (1934), 227-235; Corrigendum, ibid., 413.

\footnotetext{
Department of Mathematics

Tokyo Metropolitan University

Fukasawa, Setagaya

Tokyo 158, Japan
} 